\title{
Boundary layer flow adjacent to a permeable vertical plate with constant surface temperature
}

\begin{abstract}
The effects of suction/injection on the laminar boundary layer flow adjacent to a vertical wall with constant surface temperature are considered. The governing partial differential equations are first transformed into ordinary differential equations before being solved numerically by a finite difference method. Results for the skin friction coefficient, local Nusselt number, velocity profiles as well as temperature profiles are presented for different values of the governing parameters. It is found that the solution was unique for the assisting flow, while dual solutions exist for the opposing flow. The results indicate that the range of known dual solutions increases with suction and decreases with injection.
\end{abstract}

Keyword: Boundary layer; Dual solutions; Heat transfer; Suction/injection 